\title{
Effects of early common features on form perception
}

\author{
THOMAS SANOCKI \\ University of South Florida, Tampa, Florida
}

\begin{abstract}
Recognizing forms may involve a contingency in which later processing is modified, depending on the results of early analyses. This hypothesis can be distinguished from feature models, in which features (including early global features) accumulate over time. In four experiments, shape primes were presented briefly, followed immediately and in the same location by a similarly or differently shaped target, and then a mask. Accuracy was measured with a two-alternative forcedchoice discrimination. The primes facilitated discriminations between a similarly shaped target and differently shaped foil, as would be expected. More important is that the primes also facilitated discriminations between similarly shaped targets and similarly shaped foils, even though the primes contained only features common to the alternatives and thus provided no discriminationrelevant information. The facilitation effect was constant over variations in the size of the target set, the type of mask, and the type of baseline condition. This result is consistent with the idea of early-to-late contingencies in processing but was not predicted by feature models.
\end{abstract}

The problem of recognizing familiar patterns and objects presents a difficult challenge for the visual system, because such forms may take on an infinite variety of appearances, varying in detail, structure, size, and orientation. Processing strategies that require the matching of all possible combinations of features against possible instantiations of known forms seem implausible because of the computational costs. One promising alternative approach is to assume that forms can be recognized from a minimal set of features that remain invariant across instances or viewpoints (see, e.g., Biederman, 1987; Gibson, 1969). In this type of approach, recognition can be modeled as a passive, bottom-up process in which features extracted in parallel activate higher level representations of form.

However, recent evidence indicates that the perceptual system is sensitive to differences between instances (see, e.g., Jacoby, Baker, \& Brooks, 1989; Sanocki, 1987, 1988) and viewpoints (see, e.g., Jolicoeur, 1985; Palmer, Rosch, \& Chase, 1981). Effects of information about instances and viewpoints support more complex models that postulate the use of information beyond minimal sets of features, or processes more complicated than passive feature accumulation (see, e.g., Sanocki, 1987; Ullman, 1989). Such richer processes or representations may be

This research was supported by a Research and Creative Scholarship Award from the University of South Florida. Thanks are extended to Asher Cohen, Lester Krueger, Sandy Schneider, and an anonymous reviewer for insightful comments on a draft of this paper; to Gregg Oden, John Palmer, and Jay Rueckl for discussion of this research; to Chris Woodard for computer programming and discussion; and to Warren Gibson, Robert Matusky, and the Graphic Cognition Lab for help in conducting the experiments. Correspondence concerning this article should be addressed to Thomas Sanocki, Department of Psychology, BEH 339, University of South Florida, Tampa, FL 33620-8200 (e-mail: sanocki@figment.csee.usf.edu). necessary for the explication of how diverse instances are handled (see, e.g., Sanocki, 1987; Ullman, 1989). The use of additional information can increase the robustness of the recognition process (see, e.g., Oden, Rueckl, \& Sanocki, 1991) and reduce the considerable burdens on higher level vision (see, e.g., Enns \& Rensink, 1991; Treisman \& Patterson, 1984). However, the computational costs of processing additional information pose a potential problem for these types of approaches.

Computational costs may be reduced in various processing strategies. For example, in computer vision it is almost standard for computer programs to use early results to delimit later processing (see, e.g., Lowe, 1985; Stark \& Bowyer, in press). In psychology, "top-down" processing, in which prior knowledge affects perception, has been of interest for some time (see, e.g., Neisser, 1976; Norman \& Bobrow, 1976; Palmer, 1975). However, claims about top-down effects have often taken a strong form, in terms of mechanisms such as knowledgedriven hypothesis testing. For example, one hypothesis was that exposure to a scene's name (the semantic level) can expedite visual recognition of objects within the scene (see, e.g., Biederman, Teitelbaum, \& Mezzanotte, 1983; see also, e.g., Henderson, 1977; Rumelhart, 1977). Most experiments have not been consistent with top-down hypotheses (see, e.g., Biederman et al., 1983; Johnston, 1978); consequently, few current models include topdown mechanisms.

However, there are other processing strategies in which later processing is modified depending on stimulus information extracted early in processing. These strategies can reduce computational costs, but they do not require prior semantic knowledge about a form. For example, in one such strategy, early global information may be extracted from a form and used to arrive at candidate interpreta- 
tions of the form, which then constrain further analyses (see, e.g., Navon, 1977). In another strategy, the spatial location of certain features may be established relatively early in processing and used in localizing later arriving features. In the present experiments, the concern is with the general class of models in which later processing is modified, depending on early stimulus information. This general idea will be referred to here as the contingency hypothesis. Although quite general, the contingency hypothesis can be distinguished from another very successful class of models.

In the present experiments, the contingency hypothesis was distinguished from a class of bottom-up models, which will be referred to as the general feature model. Feature models have received a large amount of empirical support (see, e.g., Biederman, 1987; Keren \& Baggen, 1981; Massaro \& Friedman, 1990; Oden, 1979; Townsend \& Ashby, 1982). In the general feature model, feature information accumulates continuously and in parallel, cascading upward to activate form-level interpretations. The probability of a given response depends on the evidence for that response, relative to the evidence for all other relevant responses (Luce choice rule; Luce, 1959).

In this model, the accumulation of feature information is entirely passive, although some types of features may be extracted earlier in time than others. In particular, a number of investigators have proposed that global features are extracted before local features (e.g., Eriksen, O'Hara, \& Eriksen, 1982; Lupker, 1977; Navon, 1977; Townsend, Hu, \& Kadlec, 1988). However, in contrast with what occurs in the contingency hypothesis, later processes are not modified in the feature model; global features may preponderate early in processing, but they do not affect the efficiency of later local processing.

Several types of evidence are consistent with the general idea that the percept evolves during recognition from a global, "bloblike" form to a more detailed pattern. However, this evidence does not distinguish between contingencies involving early global information and a feature model in which global features preponderate early in processing. Recognition confusions for briefly presented patterns tend to be more frequent between patterns with similar shapes (see, e.g., Bouma, 1971; Lupker, 1979), as would be expected if global features such as shape preponderate early in processing. When same-different judgments are made under conditions of speed-stress, false same responses increase in frequency, especially for visually similar different pairs (Krueger \& Chignell, 1985). This would be expected, since similar letters would have similar global features and therefore similar percepts early in processing time. Responses based on global levels of a hierarchical form can be made faster than responses based on local levels, as would be expected if global information was extracted and used first (Navon, 1977). ${ }^{1}$ Also, Townsend and his colleagues (e.g., Townsend \& Ashby, 1982; Townsend et al., 1988) have found that the probabilities of identifying components of briefly presented patterns tend to be correlated, as might be expected if components are initially encoded within global features.
None of this evidence indicates whether or not the early processing of global features affects the efficiency of later processing.

The contingency hypothesis can be distinguished from the general feature model and an early preponderance of global features within a paradigm that will be termed integration priming. The general idea is to present shape primes briefly, followed immediately by a target and then a mask. At short durations, the prime and target will be integrated together (see, e.g., Eriksen \& Collins, 1967). The main interest is in possible facilitative effects of the prime on identification of the target. Although facilitative effects of a prime (i.e., the first stimulus in a successive pair) on a target have been examined previously (e.g., by Eriksen \& Collins, 1967; Jacobs \& Grainger, 1991; and Proctor, 1981), the present experiments are unique in that the primes contain only features that are common to the response alternatives. The reasons for using common features will be apparent below.

The paradigm is illustrated in Figure 1. On each trial, a warning signal is presented and then erased, followed by a faint prime (on priming trials), which is replaced by a clear target in the same location. The interval between prime and target is close to zero, and the prime is fainter than the target, encouraging integration of the prime with the succeeding target (cf. Turvey, 1973). After its duration, the target is replaced by the mask, and the subject is given a forced-choice discrimination between two alternatives. Accuracy is the dependent measure. In the first experiments, the target set consisted of four items $(t, f$, $e$, and $c$ ) that each fell into one of two similar-shape sets, as defined by outline. The forced-choice discriminations could be between two different-shape items (e.g., $t$ vs. $c$ ) or two similar-shape items (e.g., $t$ vs. $f$ ). Finally, as noted above, the primes contained only shape features common to both items within one or the other similarshape set (e.g., the cross in Figure 1 contains features common to the $f$ and $t$ in the stimulus set).

Of most interest are consistent-prime trials, in which the prime and target have the same shape. Naturally, a prime such as the cross would facilitate discriminations between a similar-shape target and a different-shape foil (different-shape discriminations), relative to no-prime conditions. This can be expected because the prime provides shape information relevant to the discrimination. (For example, the prime could increase the probability of identifying the target as either similar-shape item from the target set, and forced choices between similar- and

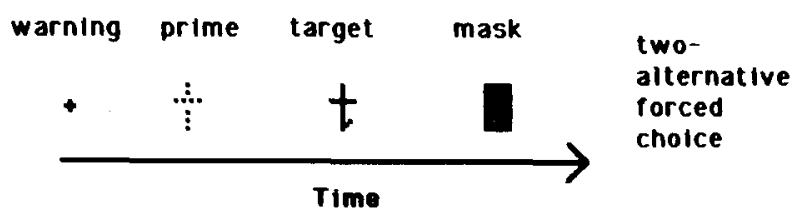

Figure 1. The sequence of events on each trial of the experiments. On no-prime trials, a blank (invisible) stimulus was presented instead of the prime. 
different-shape alternatives may be made by choosing the similar-shape item.)

The crucial question is whether or not a consistent prime facilitates discriminations between a similar-shape target and foil (similar-shape discriminations). Since the prime contains only features common to the two alternatives, the prime per se provides no discrimination-relevant information. However, the contingency hypothesis can predict facilitation, since the prime could trigger contingent processing relevant to the identity of the target or foil. In contrast, the general feature model cannot predict facilitation, because the prime provides only common features. The general feature model's prediction follows from the relative goodness rule used in most of the above-mentioned models to describe identification (Luce, 1959): The strength of one identification $\left(S_{i}\right)$ corresponds to the evidence for that identification $\left(E_{i}\right)$ relative to the sum of the evidence for all items $\left(\Sigma E_{j}\right)$,

$$
S_{i}=E_{i} / \Sigma E_{j}
$$

In a no-prime condition, the accuracy of a similar-shape discrimination should be related to the difference in strength between the alternatives $i$ and $k$,

$$
E_{i} / \Sigma E_{j}-E_{k} / \Sigma E_{j}=\left(E_{i}-E_{k}\right) / \Sigma E_{j} \text {. }
$$

A similar-shape prime would provide a fixed and equal additional amount of evidence, $P$, for each of the two relevant targets,

$\left[\left(E_{i}+P\right)-\left(E_{k}+P\right)\right] /\left(\Sigma E_{j}+2 P\right)=\left(E_{i}-E_{k}\right) /\left(\Sigma E_{j}+2 P\right)$.

Accuracy cannot be higher in the primed condition because the numerators in Equations 2 and 3 are equal but the denominator is greater in Equation 3. This holds regardless of the globality of the features. ${ }^{2}$

The exact nature of the primes may be a critical variable. Hypotheses emphasizing global-to-local processing imply that the primes should be global in nature, such as blurred or low-pass filtered versions of the targets. However, it is possible that such primes could mask targets to some degree, since they would not be exactly the same as the target. Furthermore, hypotheses involving more detailed features (such as locations) would imply that more precise primes should be used. Since the goal here was to test the class of contingency models, primes were chosen conservatively. The primes were constructed from segments of the targets that conveyed their general shape. Such primes contain many of the same global features that targets do, as well as certain local features, but they contain no information that might interfere with target identification. The importance of different types of prime information can be examined in later studies.

\section{GENERAL METHOD}

\section{Design and Stimuli}

Each experiment involved target items and primes divided into similar-shape sets; shapes (outlines) were similar within sets and different between sets. In three experiments, there were two similarshape sets of two targets and one prime each (e.g., top row, Figure 2). The primes, which were constructed from features common to all target items within their set (in their respective locations), conveyed global shape. There were six types of trials, defined by prime type and discrimination type: The primes were consistent (shape set same as the target's) or inconsistent (shape set other than the target's), or no prime was used (a blank or invisible stimulus was presented as the prime). The discriminations were either between items from the same shape set (similar shape) or between items from different shape sets (different shape).

The primes were made faint by deleting every other pixel, to encourage integration of the prime with the target. The stimuli were presented in black on white on a Macintosh SE computer. The relevant stimuli were written onto off-screen bit maps before each trial and then transferred to the screen during the trial by the same refreshsynchronized subroutine in all conditions. The refresh rate was $60 \mathrm{~Hz}$. The subjects were seated approximately $40 \mathrm{~cm}$ from the screen, so that a $t$ was $.57^{\circ}$ in height and $.33^{\circ}$ in width.

\section{Procedure}

The events on each trial were as follows: A fixation point (a “+") appeared at the center of the display area for $250 \mathrm{msec}$, followed by a blank interval of $250 \mathrm{msec}$; a priming period of 33-67 msec (depending on the experiment); a target period of approximately the same length; a masking period of $500 \mathrm{msec}$; a forced-choice display terminated by the subject's response; and either a positive (" +" for $125 \mathrm{msec}$ ) or a negative (" - " for $250 \mathrm{msec}$ ) feedback display that depended on the correctness of the response. The stimuli were centered on the same location (the letters had a common baseline). The interval between trials was $400 \mathrm{msec}$. The initial target duration varied with the experiment, and the effective duration for each subject was adjusted after each block, depending on performance during that block. Duration was adjusted upward if perfor-
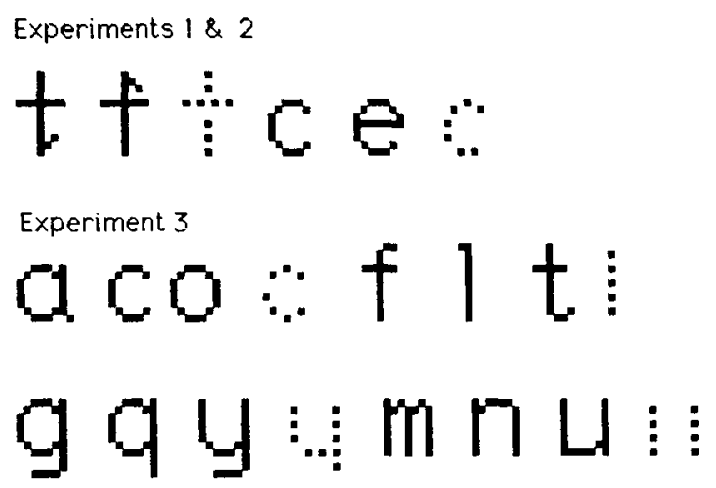

Experiment 4

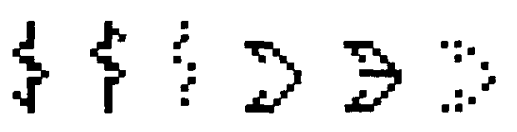

Masks

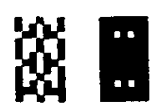

Figure 2. The targets (solid forms), primes (dotted forms), and masks (bottom row) used in each experiment. 
mance was less than $68 \%$ and downward if above $82 \%$, in steps of $17 \mathrm{msec}$, within fixed limits of 17 and $84 \mathrm{msec}$.

Blocks involved 24 trials, with equal numbers of trials in each condition. Experiments involved either 12 (Experiments 1 and 2) or 15 (Experiments 3 and 4) blocks of trials; the 1st block was treated as practice. Participants were instructed to identify the target ("darker form"), and were fully informed of the events on each trial by use of a diagram (similar to Figure 1) and a six-trial demonstration period during which prime and target intervals were increased to 250 and $334 \mathrm{msec}$, respectively.

\section{Subjects}

Different subjects participated in each experiment. They were from introductory psychology courses at the University of South Florida, and they received extra course credit for their participation.

\section{EXPERIMENTS 1 AND 2}

In Experiments 1 and 2, the targets were the four letters used in describing the paradigm above $(t, f, e, c)$. To see whether the results depended on the type of mask, a different one was used in each experiment. The magnitudes of both word-superiority and object-superiority effects vary markedly with the type of mask (Johnston \& McClelland, 1973; McClelland, 1978).

\section{Experiment I (black mask)}

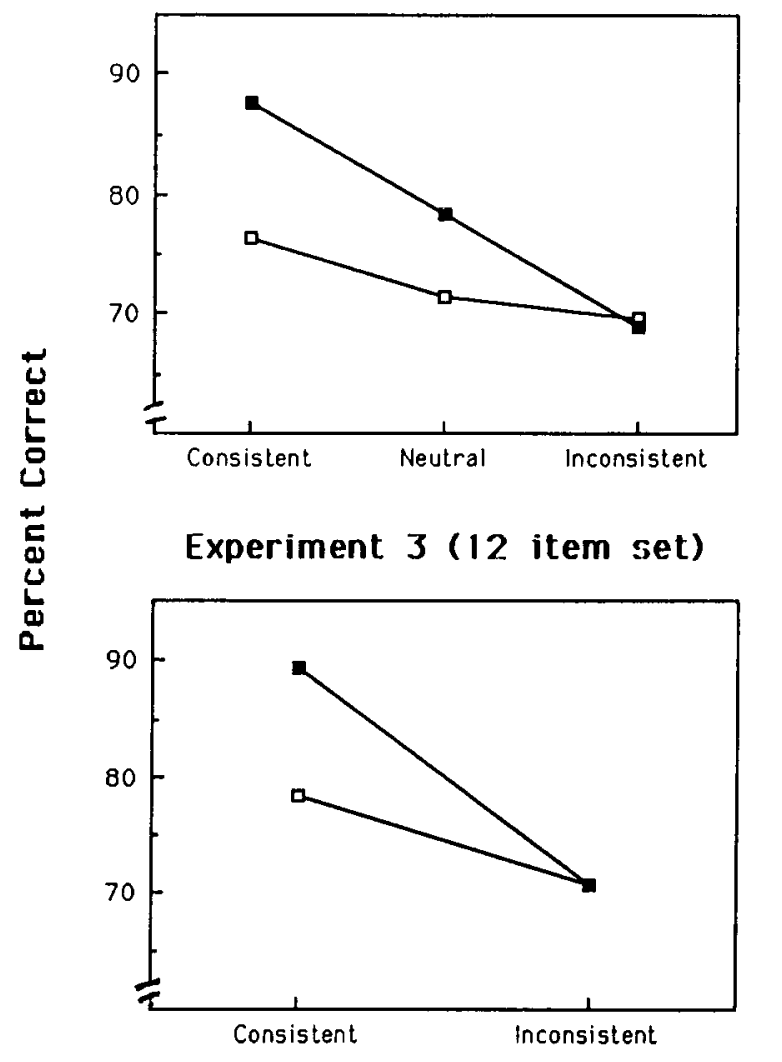

\section{Method}

The letters and primes are shown in the first row of Figure 2 . In Experiment 1, an unpatterned, "black" mask was used (second form, last row of Figure 2), and the initial target and prime durations were both $33 \mathrm{msec}$. In Experiment 2, the pattern mask was used (first form in last row, Figure 2), and the initial prime and target durations were 67 and $50 \mathrm{msec}$, respectively. Nineteen subjects provided data in Experiment 1 and 17 did so in Experiment 2. Data were discarded for 1 additional subject in Experiment 1 who failed to reach a $65 \%$ level of accuracy during the experiment.

\section{Results and Discussion}

The mean target duration at the end of Experiment 1 was $36 \mathrm{msec}$. The percent correct in each condition is shown in the upper left graph of Figure 3. There were reliable main effects of prime and discrimination type, as well as an interaction. As would be expected, discriminations were more accurate with items from different shape sets, since shape features were relevant in that type of discrimination. And, as would be expected from either the contingency hypothesis or the general feature model, priming had large effects on different-shape discriminations. Consistent primes caused facilitation relative to the no-prime condition, and inconsistent primes caused inhibition [for the main effects of discrimination type and

\section{Experiment 2 (pattern mask)}

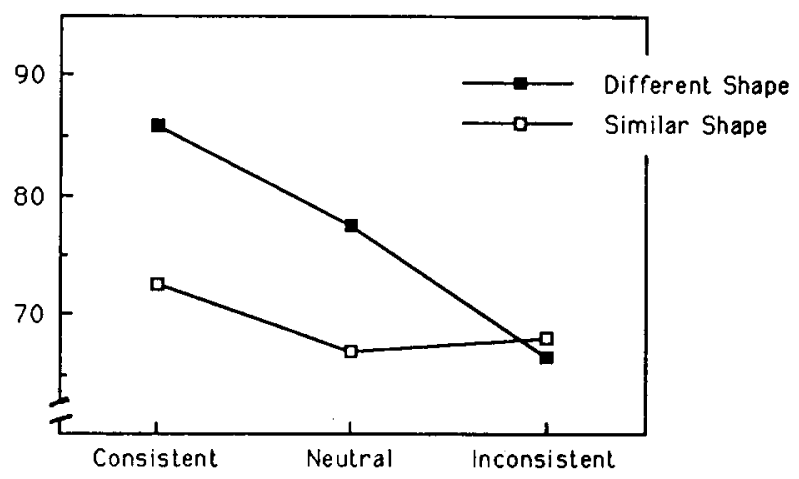

Experiment 4 (novel patterns)

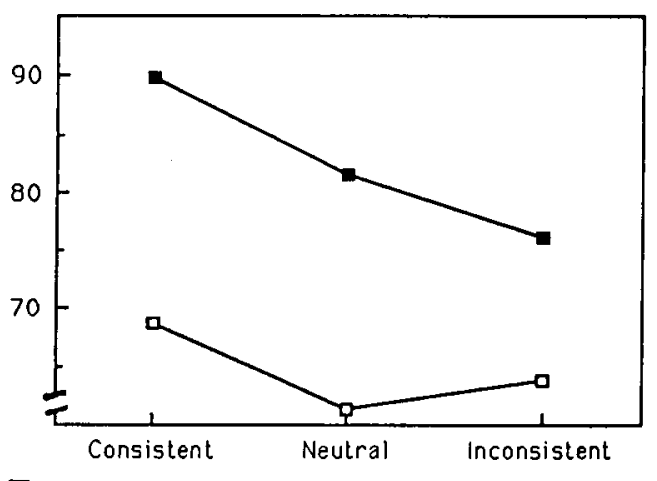

Figure 3. Percent correct discriminations as a function of discrimination type and prime type in each experiment. 
prime type, $F(1,18)=10.16, M S_{\mathrm{e}}=96.64, p<.01$, and $F(2,36)=18.21, M S_{e}=83.07, p<.001$, respectively; for the interaction, $F(2,36)=9.18, M S_{e}=38.01$, $p<.001$; for different-shape discriminations, consistent prime versus no prime, $F(1,18)=25.37, M S_{\mathrm{e}}=30.94$, $p<.001$, and inconsistent prime versus no prime, $\left.F(1,18)=15.70, M S_{\mathrm{e}}=54.32, p<.001\right]$.

The crucial question is whether consistent primes facilitated similar-shape discriminations. In fact, these discriminations were $4.9 \%$ more accurate with consistent primes than in the no-prime conditions, which is consistent with the contingency hypothesis $\left[F(1,18)=7.71, M S_{\mathrm{e}}=\right.$ $29.60, p<.05]$. There was no difference between the no-prime and inconsistent-prime conditions.

The mean target duration at the end of Experiment 2 was $56 \mathrm{msec}$. This was longer than in Experiment 1, as would be expected given that a pattern mask interferes more strongly with target identification than an unpatterned mask does. Percentages correct are shown in the upper right graph of Figure 3. Again, there were reliable main effects and an interaction. Priming effects were strongest for different-shape discriminations, with the expected facilitation and inhibition effects [for the main effects of discrimination type and prime type, $F(1,16)=$ $13.79, M S_{\mathrm{e}}=101.24, p<.01$, and $F(2,32)=13.61$, $M S_{\mathrm{e}}=91.40, p<.001$, respectively; for the interaction, $F(2,32)=7.67, M S_{\mathrm{e}}=69.77, p<.01$; for differentshape discriminations, consistent prime versus no prime, $F(1,16)=8.22, M S_{e}=75.61, p<.05$, and inconsistent prime versus no prime, $F(1,16)=10.13, M S_{\mathrm{e}}=$ $100.97, p<.01]$.

With respect to the crucial question, the contingency hypothesis was again supported by the finding that similarshape discriminations were more accurate $(5.6 \%)$ with consistent primes than with no prime $[F(1,16)=5.43$, $\left.M S_{\mathrm{e}}=49.41, p<.05\right]$. The no-prime and inconsistentprime conditions did not differ. The finding of facilitation for similar-shape discriminations in both this experiment and Experiment 1 indicates that the result is not dependent on the type of mask used in these experiments.

\section{EXPERIMENT 3}

The facilitation for consistently primed similar-shape discriminations in Experiments 1 and 2 is consistent with the contingency hypothesis. However, the generality of the finding may be limited somewhat by the fact that there were only four targets, permitting strategies based on a few distinctive features. To prevent such strategies, 12 target letters and four primes were used in Experiment 3. In this experiment, the no-prime baseline was not used; facilitation was calculated relative to inconsistently primed discriminations.

\section{Method}

There were four similar-shape sets of three letters and one prime each (rows 2 and 3 of Figure 2). The black mask of Experiment 1 was used, and the initial prime and target durations were $50 \mathrm{msec}$ each. Eighteen subjects provided the data.

\section{Results and Discussion}

The mean target duration at the end of the experiment was $\mathbf{2 5} \mathrm{msec}$. Percentages correct are shown at the lower left in Figure 3. As in the previous experiments, there were reliable main effects and an interaction, and consistent primes facilitated different-shape discriminations (relative to inconsistent primes) [for the main effects of discrimination type and prime type, $F(1,17)=23.52, M S_{e}=$ $23.26, p<.001$, and $F(1,17)=123.95, M S_{e}=25.08$, $p<.001$, respectively; for the interaction, $F(1,17)=$ $25.49, M S_{\mathrm{e}}=21.25, p<.001$; for different-shape discriminations, consistent prime versus inconsistent prime, $\left.F(1,17)=119.45, M S_{\mathrm{e}}=26.14, p<.001\right]$.

More important is that the contingency hypothesis was supported once again by higher performance $(7.7 \%)$ in the consistent-prime conditions, (this time) relative to the inconsistent-prime condition $\left[F(1,17)=26.12, M S_{e}=\right.$ $20.19, p<.001]$. This indicates that facilitation occurs with a moderately large target set; the effect is not limited to situations in which strategies based on a few distinctive features can be used. Also, the effect occurs when an inconsistent prime is used as the baseline condition, as well as with a no-prime baseline. The results are consistent with the idea that evidence from early analyses is used to modify later analyses.

\section{EXPERIMENT 4}

One important remaining issue concerns whether the facilitation effects are specific to highly overlearned stimuli such as letters or are general effects that occur during the perception of any known form, be it newly learned or highly overlearned. Effects specific to overlearned stimuli would be more consistent with processes that utilize existing knowledge, whereas general effects would be more consistent with general properties of the perceptual system such as perceptual organization. This issue can be approached by examining priming effects for novel forms.

A general comparison between familiar and novel forms involves a number of complexities and would be beyond the scope of any single experiment. In the present case, the objective was to gather initial evidence on this issue by using forms that were reasonably good and relatively novel, and that had distinctive features similar to those of the letters used in Experiments 1 and 2. Because similar distinctive features were used, any differences from the previous experiments could be attributed to the novelty of the overall patterns rather than the discriminability of the features necessary for the task.

\footnotetext{
Method

Novel forms were created by placing the distinctive features from the letters of Experiments 1 and 2 into novel contexts; the forms are shown in row 4 of Figure 2 . The design was the same as in Experiments 1 and 2. The black mask was used, and the initial prime and target durations were 50 and $33 \mathrm{msec}$, respectively. The data were from 24 subjects; data for 2 additional subjects who failed to perform above $65 \%$ were discarded.
} 


\section{Results}

The mean target duration at the end of the experiment was $47 \mathrm{msec}$. The percent correct in each condition is shown in the lower right graph of Figure 3. As in the previous experiment, there were reliable main effects of prime and discrimination type, as well as an interaction. Priming again had large effects on different-shape discriminations, with the consistent prime causing facilitation relative to the no-prime condition. The inhibitory effect of the inconsistent prime was marginal [for the main effects of discrimination type and prime type, $F(1,23)=$ $386.85, M S_{\mathrm{e}}=29.74, p<.001$, and $F(2,46)=18.21$, $M S_{\mathrm{c}}=66.65, p<.001$, respectively; for the interaction, $F(2,46)=6.05, M S_{\mathrm{e}}=47.24, p<.01$; for differentshape discriminations, consistent prime versus no prime, $F(1,23)=42.45, M S_{\mathrm{e}}=19.71, p<.001$; and inconsistent prime versus no prime, $F(1,23)=3.52, M S_{\mathrm{e}}=$ $100.39, p<.10]$.

Again, the crucial question is whether consistent primes facilitated similar-shape discriminations. As in the previous experiments, these discriminations were more accurate $(7.4 \%)$ with consistent primes than they were in the no-prime condition $\left[F(1,23)=10.27, M S_{e}=64.69\right.$, $p<.01]$. This result suggests that the contingency effect may be indicative of form perception in general, as opposed to being an effect that depends on highly overlearned representations. As in Experiments 1 and 2, accuracy with inconsistent primes did not differ from that in the no-prime condition.

One other result apparent in the data is that the overall advantage for the different-shape condition over the similar-shape condition was considerably larger in the present experiment (17.9\%) than in the previous experiments (ranging from 5.5\% to $7.4 \%$; see Figure 3 ). Since global shape features can be used in the different-shape condition but not in the similar-shape condition, this suggests that global shape features were especially important in the present experiment. Perhaps global features are more important with novel forms.

\section{GENERAL DISCUSSION}

The facilitative effects of consistent primes on similarshape discriminations are consistent with the contingency hypothesis, in which the efficiency of later processing can be increased by appropriate early information. The facilitation effect was consistent across types of masks (Experiments 1 vs. 2) and target-set size (Experiments 1 and 2 vs. 3), and it did not depend on whether performance was compared with the no-prime baseline (Experiments 1,2 , and 4) or with performance in the inconsistent condition (Experiment 3 ). The effect appears to characterize form perception in general, because the effect was obtained with novel (although letter-like) patterns in Experiment 4.

In none of the experiments was there evidence that inconsistent primes hurt similar-shape discriminations. This result might be inconsistent with some more detailed explanations of the contingency effect, but the result should be interpreted with caution because it is difficult and perhaps impossible to create a truly neutral baseline condition (Jonides \& Mack, 1984). For example, it is possible that even inconsistent primes have a facilitative alerting effect (beyond that of the warning signal) because they signal the exact onset of the target. (The warning signal always appeared between 283 and $317 \mathrm{msec}$ before the target.) This facilitative effect could offset other negative effects, resulting in performance similar to that in the noprime condition. ${ }^{3}$

The contingency hypothesis has been defined relative to the bottom-up feature model, in which stimulus information accumulates passively and early information does not modify later processing. Although the data are consistent with the idea that early information affects later processing, the mechanism by which this effect occurs is not clear.

\section{Mechanisms for Early-to-Late Contingencies}

There seem to be at least two classes of mechanisms that can explain the present effects. One class involves feature-detection processes. One possibility is that the prime could help in localizing incoming features by providing reference locations. That is, prime features might be used to establish locations to which later target features could be attached or related. The localization of features has recently been recognized as an important problem, but research is only beginning to address it (e.g., Cohen \& Ivry, 1989; Sanocki, 1991; Treisman \& Gelade, 1980). Second, the prime could help in the detection of emergent features involving relations between components, perhaps by providing partial activation of such features. Emergent or relational features have been implicated in the object-line effect (Enns \& Prinzmetal, 1984) and in the prevention of illusory conjunctions (Treisman \& Patterson, 1984), and they have also been a topic of recent interest (e.g., Pomerantz \& Pristach, 1989; Sanocki, 1991). The processes underlying localization and the detection of emergent or relational features may be related (e.g., Sanocki, 1991; Treisman \& Patterson, 1984). The present explanations seem consistent with the conclusion from Experiment 4 that the contingency effect occurs for novel forms, since the explanations do not require overlearned representations. Also, note that the prime may have mainly a facilitative effect on perception, helping with the encoding of detailed information that is consistent with it but not interfering with the encoding of other information. If this is the case, inconsistent primes would not be expected to have negative effects on accuracy.

A second class of explanations involves effects that are more top-down in nature, in that they involve effects of form-level representations on feature processing. (However, these mechanisms are not strongly top-down, in that they do not involve prior semantic knowledge.) For example, visual processing resources could become directed, on the basis of early global information, at local features of prime-consistent items (e.g., Navon, 1977). A second mechanism would be top-down feedback from the form level to the feature level, similar to that between the word and letter levels in the interactive activation model 
(McClelland \& Rumelhart, 1981). Presumably, form-level units would be activated by global features and would in turn excite additional form-consistent feature units. However, given the effects for novel forms in Experiment 4, one would have to assume that these top-down effects are caused by representations of newly learned stimuli. Although there is evidence indicating that form-level representations are created after only a single exposure to novel patterns (Kersteen-Tucker, 1991; Musen \& Treisman, 1990), it does not seem likely (at least without further evidence) that the newly created representations would cause top-down feedback.

\section{Top-Down Effects in Perception}

The extent to which perception is a "top-down" or a "bottom-up" process has been a central issue in perception and cognition over the last 20 years. As noted above, many proposals concerning top-down effects have been quite strong, involving hypothesis testing based on prior knowledge, and there is now considerable evidence against these stronger hypotheses. Most current theorists either have avoided top-down effects (e.g., Biederman, 1987) or have postulated localized effects occurring between closely related levels of analysis (e.g., McClelland \& Rumelhart, 1981).

The present effects may reflect a limited and local topdown effect, occurring within a single form. In the contingency hypothesis, the effect is stimulus-driven in that it requires (early) stimulus information; it does not result from expectancies. As Experiment 4 indicates, the effect may occur for any form that has "good" features such as lines and $45^{\circ}$ angles that form a reasonably good figure. In the latter way, the effect may be similar to the objectline effect. The object-line effect consists of the advantage in perceptibility for lines in object-like (but novel) contexts relative to lines in isolation or in less structured contexts (see, e.g., Enns \& Prinzmetal, 1984; Williams $\&$ Weisstein, 1978).

In fact, the present effects are generally consistent with facilitative effects of wholes on their parts (see, e.g., Homa, Haver, \& Schwartz, 1976; Reicher, 1969). However, the present effects also appear to be distinct from the previous results. In general, the previous "part versus whole" experiments focused on how the efficiency of processing depends on what is processed (a part, a collection of unrelated parts, or parts that form a whole). In contrast, the present experiments focus on the temporal structure of processing a single item and, in particular, on contingencies relating to the arrival of early features. The present results can complement other research on wholes and parts.

\section{The Recognition Problem}

The present results have important implications for the recognition problem because they suggest that the processing of a form may vary, depending on the results of early analyses. This increases the plausibility of models in which information beyond minimal features and processes beyond passive feature accumulation are involved in recognition (e.g., Enns \& Rensink, 1991; Oden et al., 1991; Sanocki, 1988; Ullman, 1989). More generally, the present results point to potentially important contingencies in the evolution of a percept not anticipated in currently dominant feature models. The present methods and variants of it may be used to further examine these contingencies.

\section{REFERENCES}

Biederman, 1. (1987). Recognition-by-components: A theory of human image understanding. Psychological Review, 94, 115-147.

Biederman, I., Teitelbaum, R. C., Mezzanotte, R. J. (1983). Scene perception: A failure to find a benefit from prior expectancy or familiarity. Joumal of Experimental Psychology: Leaming, Memory, \& Cognition, 9, 411-429.

Boer, L. C., Keuss, P. J. G. (1982). Global precedence as a postperceptual effect: An analysis of speed-accuracy tradeoff functions Perception \& Psychophysics, 31, 358-366.

Bouma, H. (1971). Visual recognition of isolated lower-case letters. Vision Research, 11, 459-474.

Cohen, A., IvrY, R. (1989). Ilusory conjunctions inside and outside the focus of attention. Journal of Experimental Psychology: Human Perception \& Performance, 15, 650-664.

EnNs, J. T., Pyinzmetal, W. (1984). The role of redundancy in the object-line effect. Perception \& Psychophysics, 35, 22-32.

ENNS, J. T., RENSINK, R. A. (1991). A model for the rapid interpretation of line drawings in early vision. In D. Brogan (Ed.), Visual Search II. London: Taylor \& Francis.

ERIKSEN, C. W., Coluins, J. F. (1967). Some temporal characteristics of visual pattern perception. Journal of Experimental Psychology, 74, 476-484.

Eriksen, C. W., O'Hara, W. P., Eriksen, B. A. (1982). Response competition effects in same-different judgments. Perception \& Psychophysics, 32, 261-270.

GIBSON, E. J. (1969). Principles of perceptual learning and development. New York: Appleton-Century-Crofts.

HeNDERsoN, L. (1977). Word recognition. In N. S. Sutherland (Ed.), Tutorial essays in experimental psychology (Vol. 1). Hillsdale, NJ: Erlbaum.

Homa, D., Haver, B., \& Schwartz, T. (1976). Perceptibility of schematic face stimuli: Evidence for a perceptual Gestalt. Memory \& Cognition, 4, 176-185.

Jacobs, A. M., Grainger, J. (1991). Automatic letter priming in an alphabetic decision task. Perception \& Psychophysics, 49, 43-52.

JACOBY, L. L., BAKER, J., \& BROOKS, L. (1989). Episodic effects on picture identification: Implications for theories of concept learning and theories of memory. Journal of Experimental Psychology: Leaming, Memory, \& Cognition, 15, 275-281.

Johnston, J. C. (1978). A test of the sophisticated guessing theory of word perception. Cognitive Psychology, 10, 123-153.

Johnston, J. C., McClelland, J. L. (1973). Visual factors in word perception. Perception \& Psychophysics, 14, 365-370.

JolicokuR, P. (1985). The time to name disoriented natural objects. Memory \& Cognition, 13, 289-303.

JoNiDEs, J., MACK, R. (1984). On the cost and benefit of cost and benefit. Psychological Bulletin, 96, 29-44.

KEREN, G., BAGGEN, S. (1981). Recognition models of alphanumeric characters. Perception \& Psychophysics, 29, 234-246.

KERSTEEN-TUCKER, Z. (1991). Long-term repetition priming with symmetrical polygons and words. Memory \& Cognition, 19, 37-43.

KIMCHI, R., PALMER, S. (1985). Separability and integrality of global and local levels of hierarchical patterns. Journal of Experimental Psychology: Human Perception \& Performance, 11, 673-688.

Kinchla, R. A., \& Wolfe, J. M. (1979). The order of visual processing: "Top-down," "bottom-up," or "middle-out." Perception \& Psychophysics, 25, 225-231.

Krueger, L. E., \& ChIGnell, M. H. (1985). Same-different judgments under high speed stress: Missing-feature principle predominates in early processing. Perception \& Psychophysics, 38, 188-193.

Lowe, D. G. (1985). Perceptual organization and visual recognition. Boston: Kluwer. 
Luce, R. D. (1959). Individual choice behavior. New York: Wiley.

LUPKER, S. J. (1979). On the nature of perceptual information during letter perception. Perception \& Psychophysics, 25, 303-312.

MARTIN, M. (1979). Local and global processing: The role of sparsity. Memory \& Cognition, 7, 476-484.

Massaro, D. W., Friedman, D. (1990). Models of integration given multiple sources of information. Psychological Review, 97, 225-252.

MCClelland, J. L. (1978). Perception and masking of wholes and parts. Journal of Experimental Psychology: Human Perception \& Performance, 4, 210-223.

McClelland, J. L., Rumelhart, D. E. (1981). An interactive model of context effects in letter perception: Part 1. An account of basic findings. Psychological Review, 80, 375-407.

MILLER, J. (1981). Global precedence in attention and decision. Journal of Experimental Psychology: Human Perception \& Performance, 9, $1161-1174$

Musen, G., \& Treisman, A. (1990). Implicit and explicit memory for visual patterns. Joumal of Experimental Psychology: Leaming, Memory, \& Cognition, 16, 127-137.

NAvon, D. (1977). Forest before trees: The precedence of global features in visual perception. Cognitive Psychology, 9, 353-383.

Neisser, U. (1976). Cognition and reality. San Francisco: Freeman.

Norman, D. A., Bobrow, D. G. (1976). On the role of active memory processes in perception and cognition. In C. N. Cofer (Ed.), The structure of human memory (pp. 114-132). San Francisco: W. H. Freeman.

ODEN, G. C. (1979). A fuzzy logical model of letter identification. Journal of Experimental Psychology: Human Perception \& Performance, 5, 336-352.

Oden, G. C., RueckL, J. G., \& SANockI, T. (1991). Making sentences make sense, or words to that effect. In G. B. Simpson (Ed.), Understanding word and sentence (pp. 283-304). Amsterdam: NorthHolland.

PALmer, S. E. (1975). Visual perception and world knowledge: Notes on a model of sensory-cognitive interaction. In D. A. Norman, D. E. Rumelhart, \& the LNR Research Group, Explorations in cognition (pp. 279-307). San Francisco: Freeman.

Palmer, S. E., Rosch, E., Chase, P. (1981). Canonical perspective and the perception of objects. Atrention and performance $I X$ (pp. 135151). Hillsdale, NJ: Erlbaum.

PAQuet, L., MerikLe, P. M. (1988). Global precedence in attended and nonattended objects. Journal of Experimental Psychology: Human Perception \& Performance, 14, 89-100.

Pomerantz, J. R. (1983). Global and local precedence: Selective attention in form and motion perception. Joumal of Experimental Psychology: General, 112, 516-540.

Pomerantz, J. R., \& Pristach, E. A. (1989). Emergent features, attention, and perceptual glue in visual form perception. Joumal of Experimental Psychology: Human Perception \& Performance, 15, 635-649.

Proctor, R. W. (1981). A unified theory of matching task phenomena. Psychological Review, 88, 291-326.

REICHER, G. M. (1969). Perceptual recognition as a function of meaningfulness of stimulus materials. Journal of Experimental Psychology, 81, 274-280.

Rumelhart, D. E. (1977). Toward an interactive model of reading. In S. Dornic (Ed.), Attention and performance $I V$. Hillsdale, NJ: Erlbaum.

SANOCKI, T. (1987). Visual knowledge underlying letter perception: Font-specific, schematic tuning. Joumal of Experimental Psychology: Human Perception \& Performance, 13, 267-278.

SANOCKI, T. (1988). Font regularity constraints on the process of letter recognition. Joumal of Experimental Psychology: Human Perception \& Performance, 14, 472-480.

SANOCKI, T. (1991). Intra- and inter-pattern relations in letter recognition. Joumal of Experimental Psychology: Human Perception \& Performance, 17.

STARK, L., BOWYER, K. W. (in press). Achieving generalized object recognition through reasoning about association of function to structure. IEEE Transactions on Pattern Analysis \& Machine Intelligence.

Townsend, J. T., ASHBY, F. G. (1982). Experimental test of contemporary mathematical models of visual letter recognition. Joumal of Experimental Psychology: Human Perception \& Performance, 8. 834-864.

Townsend, J. T., Hu, G. G. . KadLec, H. (1988). Feature sensitivity, bias, and interdependencies as a function of energy and payoffs. Perception \& Psychophysics, 43, 575-591.

Treisman, A., Gelade, G. (1980). A feature-integration theory of attention. Cognitive Psychology, 12, 97-136.

Treisman, A., Patrerson, R. (1984). Emergent features, attention, and object perception. Joumal of Experimental Psychology: Human Perception \& Performance, 10, 12-31.

TURVEY, M. T. (1973). On peripheral and central processes in vision: Inferences from an information processing analysis of masking with patterned stimuli. Psychological Review, 80, 1-52.

Ullman, S. (1989). Aligning pictorial descriptions: An approach to object recognition. Cognition, 32, 193-254.

WARD, L. M. (1982). Determinants of attention to local and global features of visual forms. Journal of Experimental Psychology: Human Perception \& Performance, 8, 562-581.

Williams, A., Weisstein, N. (1978). Line segments are perceived better in a coherent context than alone: An object-line effect in visual perception. Memory \& Cognition, 6, 85-90.

\section{NOTES}

1. The relevance of hierarchical form studies in the present context is questionable, however. The advantage for global levels depends on the size of the stimuli (see, e.g., Kinchla \& Wolfe, 1979) and certain other boundary conditions (see, e.g., Martin, 1979; Pomerantz, 1983). The advantage for global levels may indicate only that large forms are processed more rapidly than small forms, because the actual features in this situation have not been established (Kimchi \& Palmer, 1985; Sanocki, in press). Furthermore, the advantage for the larger (global) forms may reflect differences in the ease of allocating attention to larger stimuli, rather than differences in visual encoding (see, e.g., Boer \& Keuss, 1982; Miller, 1981; Paquet \& Merikle, 1988; Sanocki, in press; Ward, 1982). In agreement with this idea, there are advantages for larger letters in mixed-size strings of letters that appear mainly late in the time course of processing, and only at rightward positions, where attentional capacity may be limited (Sanocki, in press). But most important for the present context is the lack of relation in hierarchical stimuli between the global and local levels. Although such orthogonality is useful in the design of experiments, it contrasts with natural stimuli and makes contingencies involving early global information irrelevant.

2. Similar predictions would be derived if the target were assumed to activate features of different-shape items. (Above, on the other hand, the target was assumed to activate only features of similar-shape items, which seems a reasonable assumption.) If the target activates features of different-shape items, and if the prime provides evidence against different-shape items, the prime could have a positive effect within the general feature model, because the prime would reduce evidence in the denominator. However, because the prime also has the effect of adding evidence to the denominator (evidence for the consistent target and foil, $2 P$ ), facilitation would be predicted only if the prime's negative effect on different-shape items were greater than its positive effect on similarshape items $(>2 P)$. This seems unlikely, since different-shape items share no features with the target and therefore should not be highly activated in the first place.

3. The lack of a negative effect for inconsistent primes on similarshape discriminations might also seem inconsistent with the finding of a negative effect for inconsistent primes on different-shape discriminations. However, it is likely that different processes are operative in the two situations, since global shape features are relevant to different-shape discriminations. For example, as noted in the introduction, subjects could identify a target as either item within a shape set and use this information to make correct different-shape discriminations on many trials. However, inconsistent primes would provide evidence for the wrong response under this strategy.

(Manuscript received March 12, 1991; revision accepted for publication June 24,1991 .) 